\title{
ENFOQUE INTEGRAL DEL INGENIERO DEL SIGLO XXI: UNA REVISIÓN DE LA LITERATURA
}

\author{
Lina María Montoya Suárez¹, Jorge Andrés Cock Ramírez², Santiago Muriel Hurtado³
}

${ }^{1}$ Magister en ingeniería de software. Docente Universidad Católica Luis Amigó. Grupo de investigación SISCO. Medellín-Colombia. lina.montoyasu@amigo.edu.co ${ }^{2}$ Magister en Ingeniería con énfasis Automática. Docente Universidad Católica Luis Amigó. Grupo de investigación SISCO. Medellín-Colombia. jorge.cockra@amigo.edu.co ${ }^{3}$ Estudiante del programa de Ingeniería de Sistemas, Universidad Católica Luis Amigó. Medellín-Colombia. santiago.murielhu@amigo.edu.co

\section{RESUMEN}

Debido al crecimiento y desenvolvimiento del ingeniero del siglo XXI es fundamental conocer e identificar los diferentes enfoques en la actualidad. Este trabajo tiene como objetivo presentar los resultados de investigaciones encontrada en la revisión sistemática de la literatura, para identificar los principales enfoques de profesionales de la Ingeniería. Se concluye en seis enfoques diferentes que unidos materializan el conjunto de características propias de la actividad ingenieril, más específicamente, de quien se desenvuelven como Ingeniero del siglo XXI. Es importante mostrar en los resultados con mayor grado de detalle cada uno de estos enfoques encontrados como lo son: por competencia, social, investigación, innovación, integral, profesional que hace parte del perfil y las características profesionales.

Palabras clave: Perfil del ingeniero, enfoque general del ingeniero, competencias, competencias profesionales, competencias genéricas, competencias específicas, ingeniería, ingeniero siglo XXI.

Recibido: 16 de Agosto de 2017. Aceptado: 17 de Abril de 2018

Received: August 16th, 2017. Accepted: April 17th, 2018

\section{COMPREHENSIVE APPROACH OF $21^{\text {st }}$ CENTURY ENGINEER: A LITERATURE REVIEW}

\begin{abstract}
Due to the growth and development of the $21^{\text {st }}$ century engineer it is essential to know and identify the different approaches currently. The objective of this work is to present the results of the research found in the systematic review of the literature, to identify the main approaches of engineering professionals. It concludes in six different approaches that together materialize the set of characteristics characteristic of the engineering activity, more specifically, of those who develop as a XXI Century Engineer. It is important to show in the results with greater degree of detail each of these changes found as it is: by competition, social, research, innovation, integral, professional that is part of the profile and professional characteristics.
\end{abstract}

Keywords: Engineer profile, general engineer approach, competencies, professional competencies, generic competences, specific competencies, engineering, engineer of the XXI century.

Cómo citar este artículo: L. M. Montoya Suárez, J. A. Cock Ramírez, S. Muriel Hurtado, "Enfoque Integral del Ingeniero del Siglo XXI: Una Revisión de la Literatura", Revista Politécnica, Año 14, vol. 14, no. 26, pp. 9-18, 2018. https://doi.org/10.33571/rpolitec.v14n26a1 


\section{INTRODUCCIÓN}

Toda disciplina se respalda en teorías y conceptos que le dan soporte y finalidad para estar bien consolidada como profesión; cuando se habla de la Ingeniería no es diferente, posee unas características que la identifican, particularmente para proceder a su práctica, es fundamental conocer los principios, finalidad, estructura, gestión y fundamentos sobre los cuales se sustenta para su aplicación en la cotidianidad y en las necesidades particulares que demandan las empresas. Con respecto a esta disciplina se debe tener claro el enfoque para de esta forma comprender la labor que ha de desempeñar el ingeniero de hoy, tanto en el ámbito profesional, laboral como social, es necesario conocer su esencia, para comprender su objeto de estudio. Las personas que hoy desean convertirse en auténticos ingenieros en ocasiones no tienen un conocimiento claro de lo que es la Ingeniería en sí, desconocen al detalle el valor esencial de esta disciplina y cuál es el verdadero significado de ser ingeniero.

Para la comprensión y entendimiento de lo que significa ser ingeniero o ingeniera, para responder a la pregunta es necesario definir al profesional en ingeniería en el mundo actual. Es preciso comenzar diciendo que, el profesional en Ingeniería es un egresado de una Institución de Educación Superior o de un Instituto avalado por el ministerio de educación, para desempeñarse como profesional con el fin de responder a las necesidades actuales. Es importante saber que existen distintas definiciones de Ingeniería, sin embargo, muchas de ellas no describen con certeza lo que significa esta disciplina, ni tampoco su esencia, que la distingue de las demás disciplinas y, en especial, de aquellas que tienen elementos y propósitos muy similares, es decir:

"El ingeniero es una persona mediante sus conocimientos, experiencias, ideas y recursos (humanos y materiales) piensa y construye todo tipo de producto, con el fin de realizar proyectos o generar desarrollos, estructuras, modelos para los diferentes procesos; su objetivo fundamental es mejorar la calidad y necesidades de vida del ser humano" [1], además es "El arte de administrar los recursos de los cinco elementos de la naturaleza para su utilidad y su vivencia" [2]. Debido a la falta de claridad que se presenta algunas veces cuando se trata de la ingeniería, se busca realizar un acercamiento a aquellos elementos que identifican y definen a la persona que ejerce una profesión claramente importante que persigue en esencia un propósito tan noble como el mencionado en las definiciones anteriores.

El presente escrito pretende es desarrollar un análisis de los enfoques del ingeniero del siglo XXI, este tiene como objetivo presentar los resultados de una revisión sistemática de literatura (RSL) [3][4], la cual busca responder a la siguiente pregunta de investigación: P1. ¿Cuáles son los enfoques del ingeniero del siglo XXI? Se revisaron publicaciones en bases de datos científicas y publicaciones web especializadas para responder al cuestionamiento.

El artículo concluye que a pesar de que se ha investigado poco sobre el tema, existe evidencia científica suficiente para afirmar que el Ingeniero del siglo XXI en su campo a ejercer, es fundamental desarrollar los diferentes enfoques con integralidad; ser un Ingeniero de hoy debe ejercer los diferentes enfoques como lo son por competencia, social, investigativo, innovación, integral y profesional como un todo es decir integralmente.

\section{REVISIÓN SISTEMÁTICA DE LA LITERATURA}

\subsection{El significado de ser Ingeniero}

Para entender qué significa ser ingeniero es importante comprender qué es la Ingeniería a continuación [5]:

"Ingeniería es la habilidad de orientar los recursos y materiales del planeta, para la vida humana" el profesional desarrolla el arte de diseñar, estructurar y construir donde se prueban las esencias que se implementa en la vida cotidiana; se refiere también a la selección, aplicación de los materiales y de las fuerzas que intervienen sobre un objeto cualquiera, el objetivo esencial de la ingeniería es un agente activo que está al servicio de la humanidad. Dichas características y componentes de la ingeniería, expresan el deber ser. Además, la ingeniería tiene como razón de ser el hombre y todo lo que lo rodea, es decir, todo trabajo de ingeniería es fundamental presenciar como objetivo al día de hoy la sociedad y la humanidad en general. Con respecto a la definición anterior los ingenieros:

1. Deben evaluar sus diseños objetivamente, asegurándose de que los efectos positivos superen 
los efectos adversos y que, al hacer un balance, sus soluciones sean para el beneficio de la sociedad y del hombre en general.

2. Están siempre en contacto con todos los procesos de la activad humana.

3. Deben tomar decisiones sobre los aspectos técnicos de su especialidad.

4. Deben solucionar problemas sociales y enfrentar reacciones humanas frente a sus proyectos $y$ diseños, que constantemente se encuentran involucrados en problemas legales, económicos y sociológicos.

"La ingeniería trasciende los pensamientos humanos al punto de analizar que debe querer y por qué debe quererlo hacer en otras palabras ¿Cuál es el propósito? Esto se reconoce como una necesidad con el fin de definirse para dar solución. Por tanto, los ingenieros, hoy por hoy se están interesado en todo tiempo de cómo dar respuesta a problemas complejos y cómo reaccionar ante aquello que lo rodea" [5].

La Ingeniería, de la mano de los grandes descubrimientos científicos y los avances tecnológicos que facilitan las labores desempeñadas por los profesionales en esta área, se considera un arte, aunque también es considerada como una ciencia. Los ingenieros son artistas que, aunque siguen métodos sistemáticos y ordenados igual que los científicos, requieren libertad para crear, innovar, mejorar, estructurar, proponer, investigar, plantear, exponer, consolidar, trazar y trabajar nuevos conceptos, para el estudio de fenómenos conocidos o el rediseño de los mismos, pero bajo condiciones mejoradas. Cross afirma que la ingeniería es arte y no solamente Ciencia y Tecnología [2].

En definitiva, no basta con tener el control de los recursos, para diseñar soluciones ingenieriles a diferentes situaciones y problemáticas de la sociedad, esto debe transcender a las necesidades humanas este es el papel que debe ser considerado por un gran ingeniero, si el dominio de las ciencias básicas (matemáticas, ciencias naturales y sus aplicaciones) no viene acompañado de una gran dosis de inventiva, innovación, creatividad, calidad, gestión, consolidación, diseño, estructura y estética; permanecería como un ingeniero de catálogo que solo se limitan a emplear técnicas, métodos estandarizados de procedimientos, cálculos y especificaciones sin ejercitar la creatividad y la inventiva como parte fundamental de su qué hacer cotidiano, con respecto a lo mencionado anteriormente se ha estructurado los diferentes enfoques que debe tener presente el ingeniero de hoy.

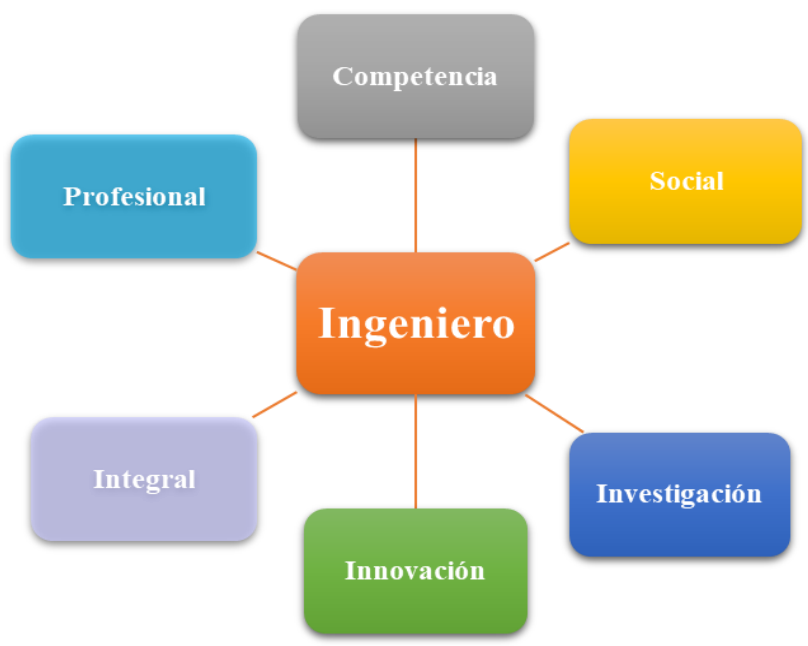

Fig 1. Enfoque general de un ingeniero del siglo XXI. Elaboración propia. Elaboración de los autores

\subsubsection{Ingeniero con enfoque en competencia.}

Las competencias profesionales del Ingeniero de hoy constituyen el punto de inicio para el diseño en el proyecto educativo, estas se definen como las prácticas cotidianas, "saber movilizar" : lo que significa construir los conocimientos o habilidades en todos los ámbitos a diferencia de ser competente, en otras palabras es adquirir la habilidad que se manifiesta en la acción; no es improvisar, es tener la capacidad de los recursos para generar ideas que ayuden no a competir sino ser competitivo, no hay competencia, habilidades, destrezas; o si los recursos no están presentes en el momento oportuno, en el momento preciso, él ingeniero debe permear los conocimientos para transformar [6]. "La transformación es una manera de movilización esto conlleva y hace que situaciones complejas lleven al ser humano a pensar y plantear los problemas para dar soluciones pertinentes, a reorganizarse en función de la situación, a colmar los vacíos y extrapolar cada situación que se presente [7].

Ser ingeniero con competencias que integra los conocimientos profesionales, destrezas, habilidades, logros en las actitudes en la actividad a desempeñar en el puesto de trabajo en un contexto 
concreto, realizando estas tareas eficaz [6]. Además, tiene un conjunto actitudes y destrezas básicas, esenciales para desempeñarse en una función específica [8]. Desempeñan un rol importante; "Las competencias genéricas o transversales, comprenden un papel importante en la formación del ingeniero en diferentes áreas en especial en la informática: permite y constituye la unidad de todas las competencias específicas donde hace sinergia haciendo diferenciador entre el saber y el saber aplicar de forma óptima y con deontología profesional" [9].

Estas se expresan aplicadas a la Ingeniería en la informática, pero sus alcances son pertinentes y resultan apropiados para la ingeniería a un nivel general. Como parte de este grupo de competencias genéricas se tienen: las competencias instrumentales, interpersonales, sistémicas. Las primeras pretenden conceder a los hombres de hoy medios y métodos que le permitan usar sus conocimientos en distintos entorno en el que se desenvuelven [9]. Las segundas favorecen el fortalecimiento del enfoque social, del que se hablará posteriormente, en especial de las habilidades comunicativas. Las últimas señalan que un ingeniero debe transcender y adquirir capacidades que permitan entender el conjunto de complejidades y así trabajar más allá de los objetivos simples e inmediatos, por ejemplo, trabajar en un contexto social y económico [9] [10].

Por consiguiente, el hecho de ubicarse en un contexto socioeconómico determinado enmarca una serie de situaciones y aspectos que son considerados para la toma de decisiones además de la repercusión de dichas elecciones en el futuro inmediato, incluso en el futuro lejano. Aun cuando es imposible hablar con exactitud, pues siempre habrá cierto grado de incertidumbre cuando se trata del futuro, si con aproximaciones que permitan establecer o determinar ese nivel de impacto [11] .

\subsubsection{Ingeniero con un enfoque social}

El enfoque social reconoce al profesional en Ingeniería como una persona que desempeña sus labores en grupos de trabajo. Por lo tanto el comportamiento ético y moral será fundamentado en la formación no sólo bastar ser ingeniero primero debe sebe ser persona con habilidades y capacidades sociales que sea indispensables para saber interactuar con grupos heterogéneos [9]. La comunicación asertiva en los grupos de trabajo debe ser primordial para lograr establecer buenas relaciones entre colegas y compañeros, solo así será posible explotar al máximo las capacidades de cada uno de los equipos. Pero este enfoque no sólo implica una relación armoniosa con grupos de personas sino también con el ambiente, al promover una eficiente y concienzuda manipulación de los recursos disponibles [12].

Como parte de la vocación creadora del ingeniero la interacción entre sus propias ideas y las de otros es innegable. "La creación como suceso emprendedor que promueve y robustece la relación humana unida a la naturaleza que nos rodea, en otras palabras con el mundo en el cual habita" [13]. Cuando se trabaja de forma cooperativa, respetando, escuchando, dando valor a las ideas de los otros es más factible alcanzar los resultados esperados y con menor frecuencia se cometen errores. Una opinión, un punto de vista diferente, puede dar perspectiva a una situación problemática que a simple vista parece no tener salida o puede también ofrecer otro camino más acertado, una solución más viable a la que en algún momento aparentaba ser la más prometedora.

\subsubsection{Ingeniero con un enfoque en investigación}

Los ingenieros no solo son responsables de la creación y el diseño de obras, dispositivos y procesos, sino que también se dedican a la dirección de su construcción, por ejemplo: un ingeniero civil que dirige la construcción de un edificio, un puente o un acueducto; un ingeniero industrial que dirige 0 administra un sistema productivo para la fabricación de automóviles, o un ingeniero eléctrico que opera o administra una planta generadora de energía eléctrica. La ingeniería implica la aplicación de los conocimientos y destrezas adquiridos para darle solución a un problema, lo cual significa que no basta con adquirir los conocimientos, habilidades y destrezas necesarios, si estos no son aplicados a la creación de soluciones a problemas propios de cada especialidad [14].

Para lograr esa aplicabilidad de los conocimientos la incesante búsqueda de nuevo conocimiento es imprescindible, cada nuevo avance tecnológico es una nueva oportunidad para el desarrollo de 
propuestas más ambiciosas y prometedoras. Todo esto sujeto a un alto sentido de responsabilidad además de respeto por la condición humana y el medio ambiente en general. Se tiene aquí un asunto que se debe tratar con cautela pues en ocasiones no se mide el impacto de los grandes avances tecnológicos o científicos, así entonces, surge la necesidad de la prudencia al momento de actuar. La constante supervisión y el respeto por las normas son claves en este sentido. "Los Comités de Ética Científica trabajan por la relación frente a los avances de la ciencia y la tecnología; pensando en el investigador de hoy transcienda las fronteras de la sociedad moderna, con el propósito investigativo como principal tarea, así lograr la optimización de los proceso y cuidar los derechos del ser humano a través del respeto a la dignidad humana" [15].

El principio de precaución (PP) sugiere: "Fundamentalmente practicar la precaución con las nuevas aplicaciones tecnocientíficas, y minimizar riesgos potenciales que permitan la implementaciones de aplicaciones ecológicos, sociales, económicos, políticos, tecnológicos y otras áreas que sean pertinentes" [16]

La investigación traza un camino evidentemente conectado con el aprendizaje y en este último elemento Gonzalo Ulloa señala "Los estudiantes que estudia de alguna manera sobre la ciencia, aprende de forma fragmentada, es decir sin lógica, trabajando la memoria y los procedimientos mecanizados, el estudiante no comprende, ni entender el porqué de las cosas, quedan con miedo frente a las ciencias presentadas de esta manera" [17]. Vale la pena mencionar aquí que la formación y el desarrollo del espíritu investigativo del estudiante será un factor preponderante en su futuro desempeño. Por esta razón conviene reestructurar como lo propone el autor la forma en la que desde la educación media se trabaja este componente investigativo [18]. Lo importante no es saber, sino saber para qué sirve lo que se sabe. Este es el problema de los doctores que tienen la Ingeniería cautiva del discurso de la Ciencia.

\subsubsection{Ingeniero con un enfoque en innovación}

Un ingeniero es, ante todo, un profesional que, aplicando la ingeniería, es decir los conocimientos, habilidades y destrezas propias de esta disciplina, busca, recomienda y da solución a un determinado tipo de problemas, expectativas y deseos de la sociedad. Normalmente su problema comienza al ser consciente de una necesidad, carencia o deseos que puede satisfacer por medio del diseño y sin dejar atrás la construcción de un dispositivo. La innovación requiere de inventiva, pero también de espíritu investigativo, de un enfoque social y del desarrollo de competencias profesionales. La capacidad para reinventarse va inmersa en este enfoque innovador del que se habla. "La innovación es la fase de un punto de partida para transformar ideas en valor fundamental de una empresa, con la finalidad de pasar en las necesidades, viabilidad, estructura, modelo, entre otros hasta la lograr la implementación de algo nuevo, novedoso e innovador que significativamente mejora un ya sea un producto, servicio, proceso y esquema" [19].

Basados en esta definición resulta natural pensar en relaciones de congruencia entre el perfil ingenieril y el enfoque de innovación, teniendo en cuenta que "en ocasiones la ingeniería se define como la aplicación e interacción de los conocimientos existentes para la solución de problemas socioeconómicos; esta constituye a la tarea de transformación de los resultados científicos en productos o procesos nuevos" [20]. Podría decirse entonces "La ingeniería establece una de las actividades más importantes en el proceso de innovación y su existencia nos permite tenerla como una condición necesaria en el desarrollo potencial y moderno para trascender cada vez más [20].

Para reforzar aún más esta idea y continuar resaltando el enfoque en innovación del profesional en ingeniería, es preciso mencionar que esta persona se encuentra inmersa en el tridente mágico que dinamiza y potencia el correcto funcionamiento del desarrollo empresarial, pues "La ciencia, la tecnología y la innovación, son conocimientos que deben formar parte de la sociedad moderna, de manera que las empresas, sin importar su tamaño, volumen, sectores y contexto de sus actividades productivas, puedan ser competentes hoy en el siglo XXI" [21]. La experiencia sumada a las herramientas que proveen la ciencia y la tecnología posibilitan entonces un óptimo nivel de progreso en la fundamentalmente necesaria, enriquecedora, revitalizadora innovación; en la dominante Sociedad del Conocimiento (Knowledge Society) [21] 


\subsubsection{Ingeniero con enfoque integral}

"Las competencias están basadas, al desempeño exitoso en una función y por lo tanto integran el saber, el hacer y el ser en un contexto presentado" [22]. El enfoque integral pretende que no sólo estén presentes los conocimientos técnicos adquiridos durante la formación profesional, sino que también se integren las diferentes competencias transversales, las cuales conforman entre sí, el ser, el saber y el hacer. Tomando la esencia del enfoque integral conviene expresarlo mejor como una acción integrar que implica movimiento, dinámica. Una dinámica que propone, como se menciona con anterioridad, movilizar los tres elementos ser, saber y hacer. "Significa precisar o determinar propósitos, valores, ética, habilidades blandas para ser utilizarlos en la cotidianidad de manera que se refleje significativamente, sin dejar atrás los principios y el servicio"[23][24]. En ocasiones se deja de lado el compromiso serio que como profesional se asume para poder satisfacer todas las obligaciones que el título demanda, limitándose únicamente a la cuestión del saber y el hacer, dejando de lado el ser [25].

El ser proporciona el desarrollo de lo que permite realmente la integralidad para alcanzar la verdadera grandeza de un profesional completo. Pero no por sí solo, pues debe existir sana convivencia entre las competencias específicas y genéricas del ingeniero, como un conjunto y no por separado. Como una visión holística de la persona y todo aquello que conforma su formación personal, académica y laboral que se complementa y se retroalimenta de forma constante sin dejar de deja de aprender nuevas cosas. A eso conduce la denominada sociedad del conocimiento como paradigma tecnológico. Otro aspecto al que hace referencia el enfoque integral es a la existencia de una aparente confrontación entre elementos supuestamente antagónicos que durante años han hecho parte de la formación de todo tipo de profesionales: saberes y competencias. Pero en realidad esta confrontación no es más que una relación de complementariedad pues "Los saberes, y el enfoque por competencias da una fuerza, evolución y transformación a las prácticas sociales, económicas y culturales a diferentes escenarios complejos"[7].

\subsubsection{Ingeniero con enfoque profesional}

El enfoque profesional hace referencia al reconocimiento del ingeniero como aquella persona capacitada para desempeñarse en el medio, de forma profesional y competente, con la obligación de dejar en alto su imagen y asumir con responsabilidad su cargo. Ser profesional implica ser realmente [26] , nuestra capacidad tecnológica no es neutral sino ambivalente así que depende de cada uno aplicar de forma responsable y con alto grado de ética todas las habilidades afines a su especialidad. Adicional a esto, refiriéndose a un contexto caracterizado por la globalización, en el cual se mundializan posibilidades, pero también problemáticas los autores también mencionan: "Este contexto de ser profesional en el siglo XXI atañe indisolublemente a la ciencia, tecnología, comunicación, planteando cuestionamientos si todo lo técnicamente posible o lo económicamente rentable es humanamente deseable hoy por hoy" [26]. Es una cuestión de responsabilidad social y profesional el hecho de determinar qué tan coherentes son las funciones del cargo (cualquiera que este sea) con el código de ética profesional que enmarca la labor desempeñada por los profesionales en la ingeniería o con el reglamento interno de la compañía [27][28].

Lo anterior obliga al profesional a ubicarse en un contexto y un momento dados para responder de la mejor manera ante las funciones que debe desempeñar y los objetivos que dichas funciones persiguen. Poco se ha hablado hasta ahora de la gran influencia que tienen en la formación profesional del ingeniero dos elementos que denominan en su estudio como: creencias de competencia y creencias de valor (competence beliefs and value beliefs) [14]. Acerca de cómo influye la identidad personal en la aspiración de obtener un título de ingeniería. No es un secreto que algunos de los rasgos de la personalidad de los estudiantes pueden llegar a ser verdaderos catalizadores de un destacado rendimiento académico y laboral [29]. Sin duda un mejor entendimiento de estos aspectos desde la perspectiva de los estudiantes de ingenierías contribuiría notoriamente a desarrollar mejoras en los diferentes programas de formación relativos a la ingeniería. $Y$ es precisamente este camino el que sigue el estudio realizado [30] [31]. 


\section{METODOLOGÍA}

Se empleó la metodología de la revisión sistemática de literatura (RSL), revisión sistémica, auditable y rigurosa que busca dar respuesta a una pregunta de investigación tomando como referencia investigaciones científicas realizadas con anterioridad. La pregunta de investigación considerada en este trabajo fue: P1 ¿Cuáles son los enfoques del ingeniero del siglo XXI? Se revisaron artículos, artículos de conferencias, libros y publicaciones web especializadas. Las bases de datos empleadas para la búsqueda de información fueron: Scopus, Digitalia, IEEE y Science Direct,

Las palabras claves de búsqueda fueron obtenidas a partir de los títulos de artículos y libros especializados para la selección de las fuentes primarias. El rango de fechas de búsqueda estuvo comprendido desde el año 2005 hasta el 2017. Se conformaron cadenas de búsqueda con las combinaciones de las palabras: "Engineer profile", "general engineer approach", "professional competencies engineer", "generic specific competencies engineering" en el título, resumen y palabras clave.

Las cadenas de búsqueda utilizadas fueron:

(1) (TITLE-ABS-KEY (Engineer profile) AND TITLEABS-KEY (general engineer approach $>2005$.

(2) (TITLE-ABS-KEY (Engineer profile) AND TITLEABS-KEY (professional competencies engineer)) > 2005.

(3) (TITLE-ABS-KEY (Engineer profile) AND TITLEABS-KEY (generic specific competencies engineering)) $>2005$.

(4) (TITLE-ABS-KEY (Engineer profile) AND TITLEABS-KEY (engineer of the 21st century)) > 2005.

Al hacer la revisión sistemática para la identificación y clasificación; lo encontrado en la búsqueda, se obtuvo así un primer conjunto de estudios primarios.

Tabla 1. Estudios Primarios Obtenidos

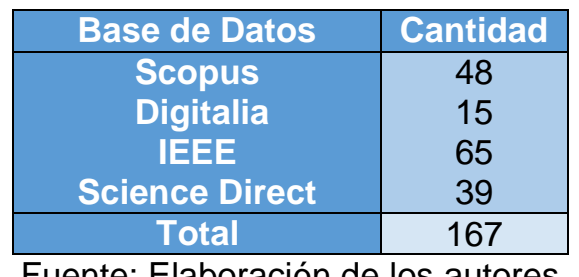

Fuente: Elaboración de los autores
De los resultados de la búsqueda se seleccionaron 26 publicaciones de las bases de datos científicas consultadas (Tabla 1).

\section{DISCUSIÓN}

El ingeniero del Siglo XXI debe estar al servicio de la humanidad y no para el uso de destruir y perjudicar al ser humano y su entorno es decir debe transcender en su profesión las necesidades cotidianas con el propósito de aportar soluciones a problemas complejos a continuación se describe lo que se identificó en la revisión sistemática.

Los Ingeniero de hoy:

1. Emplean recursos y dinámicas de la naturaleza en la creación y el diseño de soluciones a problemas de su especialidad $y$, por consiguiente, son responsables de su buena utilización y conservación [5][32] [14] [20].

2. Es primeramente humano además no se debe perder de vista que trabaja con personas, donde las relaciones interpersonales son fundamentales [9] [12] [13].

3. Buscan respuestas que solucione problemas actuales [32] [14] [18] [20].

4. Utilizan los materiales, herramientas, recursos y las fuerzas de la naturaleza (representados en los principios y las leyes de la física, la química, la biología y demás áreas) [2] [13].

5. Deben reconocer las fuentes de materiales y de energía, para ocuparse no solamente de su utilización, sino también de su conservación [14].

6. Tienen la responsabilidad de buscar soluciones que sean eficaces desde el enfoque de la energía y explorar nuevas fuentes de la misma para sustituir las que se estén agotando [14][31].

7. Deben buscar, además, soluciones que sean económicas, es decir tener en cuenta la relación costo-beneficio [18] [23] [27] . 
8. Es integral en las diferentes dimensiones donde es un ser que hace y que sabe hacer [22][25][27][28].

9. Se prepara para ser formado, competente, innovador, investigativo y sobre todo un profesional humanístico[7][9][12][14].

10. Lo importante no es saber, sino saber para qué sirve lo que se sabe y como potencializa lo que sabe [6] [9] [18].
En la tabla 2 se evidencia una síntesis del enfoque analizados en la revisión de la literatura que debe tener los Ingenieros del siglo XXI, con las características más relevantes para la contribución a la formación y desempeño del profesional.

Tabla 2. Enfoque general del ingeniero.

\begin{tabular}{|c|c|}
\hline Enfoque & $\begin{array}{c}\text { Características más relevantes } \\
\text { Contribución a la formación y desempeño del profesional en Ingeniería. }\end{array}$ \\
\hline Competencia & $\begin{array}{l}\text { Todo tipo de habilidades y destrezas que contribuyen al correcto desempeño de las } \\
\text { funciones en el campo laboral. Su clasificación se realiza por grupos de competencias que } \\
\text { pueden ser específicas o genéricas. }\end{array}$ \\
\hline Social & $\begin{array}{l}\text { Prioriza el trabajo en equipo, fomenta las habilidades comunicativas al interior de los } \\
\text { grupos de trabajo. Destaca la relación entre hombre y medio ambiente, promueve la } \\
\text { responsabilidad y el carácter social del ingeniero. }\end{array}$ \\
\hline Investigación & $\begin{array}{l}\text { Anima el espíritu investigativo bajo una constante supervisión que garantice el respeto de } \\
\text { las normas establecidas. El principio de precaución y la participación de los comités de } \\
\text { ética son algunos de los entes de vigilancia presentados. }\end{array}$ \\
\hline Innovación & $\begin{array}{l}\text { Declara que en la Sociedad del conocimiento la ingeniería representa una parte } \\
\text { importante en el proceso de innovación tecnológica y por consiguiente en el desarrollo de } \\
\text { la productividad en el mundo empresarial. }\end{array}$ \\
\hline$\overline{\text { Integral }}$ & $\begin{array}{l}\text { Propone el enfoque integral como elemento dinamizador entre el ser, el saber y el hacer. } \\
\text { Invita al ensayo de una mirada holística sobre los elementos que soportan el concepto de } \\
\text { ser un profesional completo. }\end{array}$ \\
\hline Profesional & $\begin{array}{l}\text { Enaltece la responsabilidad de la labor desempeñada por el ingeniero al poner en } \\
\text { evidencia que el aprovechamiento de la capacidad tecnológica humana debe hacerse de } \\
\text { forma consciente y responsable pues de ella se derivan elementos muy constructivos o } \\
\text { destructivos para la condición humana. }\end{array}$ \\
\hline
\end{tabular}

Fuente: Elaboración de los autores 


\section{CONCLUSIONES}

Se destaca la formación integral del ingeniero del siglo XXI y su influencia en el desempeño personal y profesional, a partir del desarrollo de los diferentes puntos focales para lograr identificar factores clave en la estructura del perfil profesional del ingeniero. A raíz de este acercamiento la ciencia y la tecnología cobran especial relevancia como fuentes de constante renovación para la actividad ingenieril, por consiguiente, ambas son determinantes para la definición de una identidad particular que se forja en el desarrollo cultural, económico, social y sustentable de la sociedad de hoy; todo esto en beneficio de la propia condición humana.

Los puntos focales no sólo se aprovechan en el ámbito laboral, sino que son preparados, formados y potenciados en todo el proceso de formación en su ciclo vital del proceso académico. La idea de un profesional integral que pueda ejercer toda su capacidad en la sociedad y que pueda suplir desde varios puntos de vista las necesidades que se presentan, en un mundo en el que está presente un panorama social, económico, político, cultural y tecnológico cambiante, conlleva a un alto nivel de expectativa en la construcción de un futuro mejor.

\section{REFERENCIAS BIBLIOGRÁFICAS}

[1] G. Aquiles, Introducción a la ingeniería : la tecnología, el ingeniero y la cultura, Editorial. Buenos Aires, Argentina, 2014.

[2] C. Hardy, Ingenieros y las Torres de Marfil. 1998.

[3] B. Kitchenham, O. P. Brereton, D. Budgen, M. Turner, J. Bailey, and S. Linkman, "Systematic literature reviews in software engineering a systematic literature review," Inf. Softw. Technol., vol. 51, no. 1, pp. 7-15, 2009.

[4] M. Staples and M. Niazi, "Experiences using systematic review guidelines," J. Syst. Softw., vol. 80, no. 9, pp. 1425-1437, 2007.

[5] O. G. Ortiz, M. Elias, and V. Rozo, Introducció $n$ a la ingeniería: Una perspectiva desde el currículo en la formación del ingeniero. Ecoe Ediciones, 2016.

[6] L. L. C, "Gestión de las Competencias: Cómo Analizarlas, Cómo Evaluarlas, Cómo Desarrollarlas," Ediciones Gestión, 2000.

[7] P. Perrenaud, "Construir las competencias, ¿es darle la espalda a los saberes?," Red U. Rev. Docencia Univ., no. Número monográfico I. Formación basada en competencias (II), pp. 1-8, 2008.

[8] G. Le Boterf, "Gestión de las competencias," Barcelona Editor. Gestión, 2002.

[9] J. Aquilino et al., "Definición de competencias específicas y genéricas del Ingeniero en Informática," pp. 1-9, 2006.

[10] M. A. C. Gutiérrez and J. V. B. Olvera, "LOS ESTUDIANTES APLICANDO EL PERMA EN LA FORMACIÓN DE INGENIEROS POR COMPETENCIAS EN MÉXICO," ANFEI Digit., no. 6, 2017.

[11] S. Tobón, "Aspectos basicos de la formacion basada en competencias.," Talca Proy. Mesesup, pp. 1-16, 2006.

[12] B. Lazzarini and A. Pérez-Foguet, "Profiling research of the engineering academics who successfully promote education in Sustainable Human Development," J. Clean. Prod., pp. 1-15, 2017.

[13] W. de J. Gómez Acevedo and D. H. Bedoya Ortiz, "La responsabilidad cocreadora del ingeniero," Revista Académica e Institucional de la UCPR, no. 78, pp. 107123, 2007.

[14] L. R. Vega-González, "La educación en ingeniería en el contexto global: propuesta para la formación de ingenieros en el primer cuarto del Siglo XXI," Ing. Investig. y Tecnol., vol. 14, no. 2, pp. 177-190, 2013.

[15] I. Acevedo Pérez, "Aspectos éticos en la investigación científica," Cienc. y enfermería, vol. 8, no. 1, pp. 15-18, 2002.

[16] H. Lacey and L. Alvarenga, "Los valores de la ciencia y el papel de la ética en la ciencia," Real. Rev. Ciencias Soc. y ..., pp. 241-246, 2008.

[17] G. Ulloa, "¿Qué pasa con la ingeniería en Colombia?," Ing. y Soc., vol. 2, pp. 38-41, 2010.

[18] B. Motyl, G. Baronio, S. Uberti, D. Speranza, and S. Filippi, "How will Change the Future Engineers' Skills in the Industry 4.0 Framework? A Questionnaire Survey," Procedia Manuf., vol. 11, no. June, pp. 1501-1509, 2017.

[19] F. G. González, "CONCEPTOS SOBRE INNOVACIÓN," 2012.

[20] I. Fernández de Lucio, "Ingeniería e innovación * 1.," 2006.

[21] O.E.A, Ciencia, Tecnología, Ingeniería e Innovación para el Desarrollo, Segunda ed. 
Washington, D.C: OEA, 2005.

[22] L. J. Tirado et al., "Competencias profesionales: una estrategia para el desempeño exitoso de los ingenieros industriales," Rev. Fac. Ing. la Univ. Antioquia, vol. 40, pp. 123-139, 2007.

[23] N. Bessis, X. Zhai, and S. Sotiriadis, "Service Oriented System Engineering," Futur. Gener. Comput. Syst., vol. 80, pp. 211-214, 2018.

[24] K. Blanchard and M. O. Connor, Administración por valores, Grupo Norm. Bogotá, 1997.

[25] E. L. Ingeniero, C. O. N. Conciencia, U. N. A. Posibilidad, P. El, and D. Sostenible, "Engineer With Social Conscience .," vol. 19, no. 2000, pp. 25-38, 2015.

[26] J. M. G. Galo Bilbao, Javier Fuertes, "Ética para ingenieros," S. A. Editorial Desclée de Brouwer, Ed. Bilbao, 2006, p. 303.

[27] R. A. Pérez Gómez, A. A. Gamboa Suárez, and C. A. Hernández Suárez, "La ética en la formación del ingeniero de minas: representaciones sociales de actores educativos," Rev. Tecnura, vol. 19, no. 44, pp. 201-208, 2015.

[28] L. Rivas and J. L. UNEFA, "Conciencia Histórica Social," Extr. http//es. scribd. com/doc/34498728/Conciencia-HistoricaSocial.[Links], 2002.

[29] K. C. Ferreira and P. G. Lima, "Proyecto tuning América Latina en las universidades brasileñas: características y ámbitos en el área de la educación," Paradígma, vol. 34, no. 1, pp. 083-096, 2013.

[30] ABET, "ABET," ANNUAL REPORT For Fiscal Year Ending September 30, 2014, 2014. [Online]. Available: http://www.abet.org/aboutabet/publications/annual-report-archive/.

[31] F. M. Palma Lama, E. A. Miñán Ubillús, and I. de los Ríos Carmenado, "Competencias genericas en ingeniería: un estudio comparado en el contexto internacional," Actas del XV Congr. Int. Ing. Proy., pp. 2552-2569, 2011.

[32] C. Selinger, Stuff you don't learn in engineering school. Wiley-Interscience, 2004. 